\title{
The Science of Fat Grafting
}

\author{
Linden Shih, BS ${ }^{1}$ Matthew J. Davis, BS ${ }^{1}$ Sebastian J. Winocour, MD, MSc, FACS ${ }^{1}$ \\ ${ }^{1}$ Division of Plastic Surgery, Michael E. DeBakey Department of \\ Surgery, Baylor College of Medicine, Houston, Texas \\ Address for correspondence Sebastian J. Winocour, MD, MSc, FACS, \\ Division of Plastic Surgery, Michael E. DeBakey Department of \\ Surgery, Baylor College of Medicine, 1977 Butler Blvd., Suite E6.100 \\ Semin Plast Surg 2020;34:5-10. \\ Houston, TX 77030-2399 (e-mail: Sebastian.Winocour@bcm.edu).
}

Abstract
Keywords
- autologous fat
grafting
- adipocyte
regeneration
- adipogenesis
- adipose-derived stem
cells
- fat graft survival

Autologous fat grafting has become a popular and well-established technique used by plastic surgeons in a variety of aesthetic and reconstructive procedures. An understanding of the basic science principles underlying fat grafting is crucial to explaining its extensive utility for soft tissue rejuvenation, volume augmentation, and body contouring-and the unpredictable fat resorption rates after grafting that pose a significant challenge for plastic surgeons. While the scientific principles of fat grafting can theoretically be exploited to optimize fat grafting techniques and increase fat tissue survival, a consensus has yet been established as to the best practices for this procedure. This review discusses the biology of adipose tissue and the scientific principles behind its behavior and survival in autologous fat grafting.
Autologous fat grafting, or the surgical transfer of adipose tissue from one area of the body to another, was first described by the German surgeon Gustav Adolf Neuber in 1893 after he successfully transplanted fat from the forearm to fill an atrophic scar on the face. ${ }^{1-3}$ Despite several early successes, subsequent reports of varying levels of graft failure prevented the technique from being widely accepted until the $1980 \mathrm{~s}^{2,4}$ Since then, positive reports regarding the safety and efficacy of autologous fat transfer have helped to repopularize the technique. Today, autologous fat grafting is widely used by plastic surgeons in a variety of reconstructive and aesthetic procedures. ${ }^{4,5}$ Clinical applications of this technique continue to expand and include many contouring, augmenting, and rejuvenating procedures, as well as the treatment of radiation damage, breast capsular contracture, scar treatment, posttraumatic deformities, congenital anomalies, burn injuries, and soft tissue defects. ${ }^{4-6} \mathrm{~A}$ recent survey showed that around $80 \%$ of plastic surgeons have used fat grafting in their practice. ${ }^{4}$ The popularity of autologous fat grafts is because of not only its utility in volume replacement and tissue quality improvement but also its low cost, low donor-site morbidity, and high levels of accessibility, availability, and biocompatibility. ${ }^{1,5}$

Despite the widespread use of autologous fat transfer, the issue of unpredictable fat resorption rates remains a major limitation of this technique. Resorption rates of grafted adipose tissue have been reported to range from 20 to $90 \%$, leading to variable reduction of total implanted volume. ${ }^{1,7}$ Resorption often results in the need for repeated procedures and, in some cases, poor aesthetic outcomes, making the achievement of lasting results following fat grafting a significant challenge. ${ }^{2,5,8}$

Wide procedural variation in fat grafting is thought to contribute to this vast difference in resorption rates. Despite much investigation into all the steps of fat grafting, there is currently still no universal protocol for this procedure. ${ }^{2,4} \mathrm{To}$ better understand fat grafting outcomes, the biological principles of adipose tissue and its mechanisms for survival in autologous fat grafting have also been extensively investigated. $^{2,9}$ A thorough understanding of the basic science behind autologous fat grafting is important to establish best practice guidelines, facilitate graft survival, and optimize procedural outcomes.

\section{Adipose Tissue Biology}

\section{Types}

Adipose tissue is generally classified into two types: thermogenically active brown adipose tissue (BAT) and energy-storing white adipose tissue (WAT). ${ }^{6}$ BAT was previously thought to exist only in infants and small mammals, but in the past decade, studies have also shown the presence of metabolically
Issue Theme Fat Grafting in Plastic Surgery; Guest Editor: Sebastian J. Winocour, MD, MSc, FACS
Copyright $\odot 2020$ by Thieme Medical Publishers, Inc., 333 Seventh Avenue, New York, NY 10001, USA. Tel: +1(212) 760-0888.
DOI https://doi.org/ 10.1055/s-0039-3402073. ISSN 1535-2188. 
active BAT in adults. ${ }^{6}$ In humans, brown fat is distributed throughout the cervical, supraclavicular, axillary, paravertebral, mediastinal, and upper abdominal regions. ${ }^{10}$ In contrast WAT, the predominant type of fat in humans, is located either viscerally or subcutaneously. ${ }^{11,12}$ It is subcutaneous WAT that is used for autologous fat grafting in plastic and reconstructive procedures and is the main focus of this review. ${ }^{6}$

\section{Composition}

White adipose tissue has two main components: mature adipocytes and the stromal vascular fraction (SVF). ${ }^{7}$ The SVF is a heterogeneous cell population composed of endothelial cells, smooth muscle cells, pericytes, leukocytes, fibroblasts, mast cells, preadipocytes, and multipotent adipose-derived stem cells (ASCs). ${ }^{8,9}$ While cellular components of the SVF make up nearly $50 \%$ of the total adipose tissue cell number, adipocytes make up $90 \%$ of the total adipose tissue volume. ${ }^{9}$ An extracellular matrix connects adipocytes and contributes to the formation of fat lobules in adipose tissue. ${ }^{12}$

\section{Adipogenesis}

Adipocytes are derived from ASCs. $^{7}$ The development of a mature adipocyte occurs in two main phases. First is the determination phase, in which the ASC commits to the adipocyte lineage, becoming a pre-adipocyte. ${ }^{7}$ At this point, the preadipocyte can no longer differentiate into other cell types. Second is the terminal differentiation phase, in which the pre-adipocyte accumulates lipid in its cytosol and becomes a mature adipocyte. ${ }^{7}$ As we age, the capacity of preadipocytes to differentiate into fully functional mature adipocytes declines. ${ }^{13}$

\section{Adipocyte Characteristics}

The size of a mature human adipocyte ranges from 50 to $150 \mu \mathrm{m}$ in diameter, and these cells can survive for up to 10 years. ${ }^{14}$ Mature adipocytes are extremely fragile and have a low tolerance for mechanical trauma and ischemia. ${ }^{7}$ Preadipocytes, which have minimal metabolic activity and are 20 times smaller than mature adipocytes, have a much higher tolerance for traumatic and ischemic insults. ${ }^{1,7}$

\section{Vascularity of Adipose Tissue}

Adipose tissue is highly vascularized, with each and every adipocyte attached to a capillary network that allows for the efficient exchange of metabolic products. ${ }^{7,14}$ Due to its high capillary density and low oxygen consumption rate, subcutaneous adipose tissue has the highest tissue partial oxygen tension ( $\mathrm{ptO}_{2}: 40-60 \mathrm{~mm} \mathrm{Hg}$ ) among all the internal organs. ${ }^{14}$ As adipose tissue expands and regresses in size throughout life, there must be parallel growth of its capillary network. ${ }^{7}$

\section{Regenerative and Proliferative Potential of Adipose Tissue}

Adipose tissue was once thought to be an inert substance used solely for energy storage, but more recent research has shown it to have significant proliferative and regenerative potentials. ${ }^{5,9}$ Much of the regenerative potential of adipose tissue is because of the ASCs of the SVF. ${ }^{1,5,7,8}$ Adipose tissue contains the highest percentage of adult stem cells of any tissue in the body, even higher than that of bone marrow. ${ }^{1,7}$ There exist as many as 4,500 ASCs per milliliter of fat compared with only 100 to 1,000 stem cells per milliliter of bone marrow. ${ }^{1}$ ASCs achieve their regenerative effects through paracrine secretion of cytokines and growth factors, with higher levels of secretion occurring in hypoxic conditions. ${ }^{1,5}$ These factors include angiogenic cytokines such as vascular endothelial growth factor (VEGF), hepatocyte growth factor (HGF), fibroblastic growth factor 2, and basic fibroblast growth factor, as well as hematopoietic cytokines such as granulocyte colony-stimulating factor and granulocyte-macrophage colony-stimulating factor. ${ }^{5,15}$ Together, these growth factors affect the differentiation of stem cells, induce angiogenesis, stimulate tissue remodeling, and promote wound healing. ${ }^{1}$ Mature adipocytes themselves have the ability to dedifferentiate into ASCs and then redifferentiate into adipocytes or other cell types such as osteocytes. ${ }^{1,8}$ ASCs are the main cell population responsible for adipocyte regeneration. ${ }^{14}$ ASCs have the ability to undergo multilineage differentiation into adipocytes and vascular endothelial cells, as well as other tissue types such as bone, cartilage, skeletal muscle, nerves, and skin. ${ }^{1,14}$ A small subset of ASCs, termed adipose-multilineage-differentiating stress-enduring (Muse) cells, may have even more potency and are thought to contribute to the ability of ASCs to differentiate into endodermal and ectodermal derived tissue such as skin and nerves. ${ }^{14,16}$ In addition to multipotency, the density of ASCs in the tissue also contributes to the potential for adipose tissue regeneration. ${ }^{14}$ Thus, ASC-deficient tissues, such as irradiated or chronically inflamed tissues, exhibit impaired adipose remodeling and expansion. ${ }^{14,17}$ Given the extensive angiogenic and regenerative properties of ASCs, there is much interest in the inclusion of concentrated levels of ASCs in fat grafts. ${ }^{1}$

\section{Adipose Tissue in Fat Grafting}

\section{Graft Revascularization}

When adipose tissue is grafted, it is transferred in an avascular fashion and must revascularize to survive. ${ }^{5,9}$ Fat graft revascularization is largely promoted by perivascular ASCs that release angiogenic factors in response to ischemia (-Fig. 1) $\cdot^{2,14}$ To achieve proper graft revascularization, each fat graft droplet $(G)$ must interact with a capillary recipient site $(R)$ in a $1: 1$ ratio to

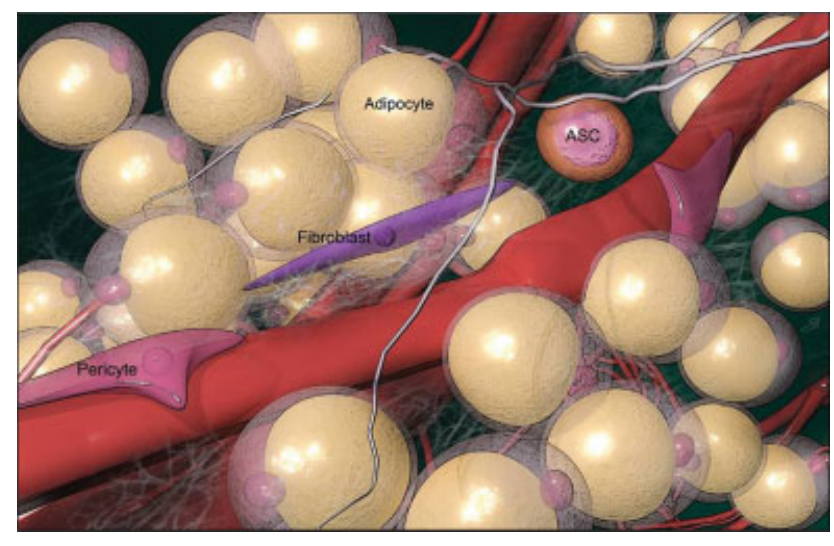

Fig. 1 Adipose-derived stem cells located perivascularly. 


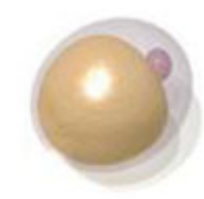

1 Fat droplet (G)

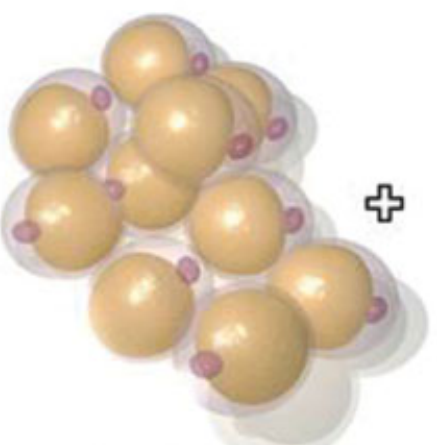

10 Fat droplets (G)

\section{\&}

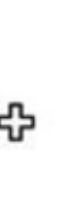

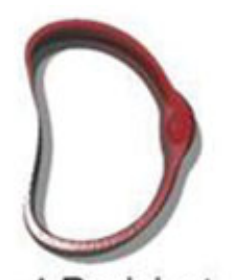

1 Recipient capillary $(R)$

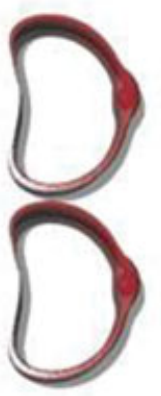

2 Recipient capillaries ( $R$ )
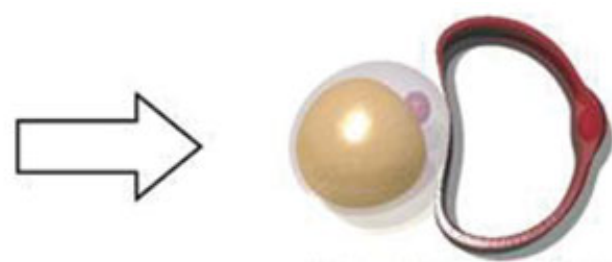

1 Surviving graft (G)

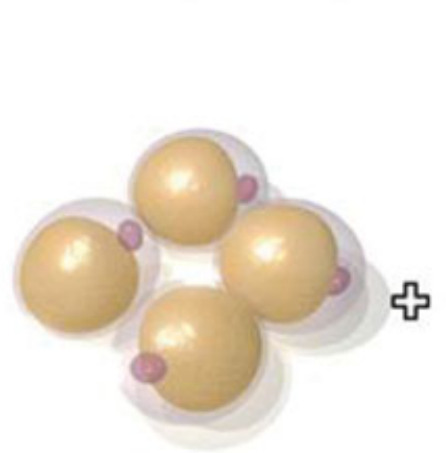

4 Fat droplets (G)
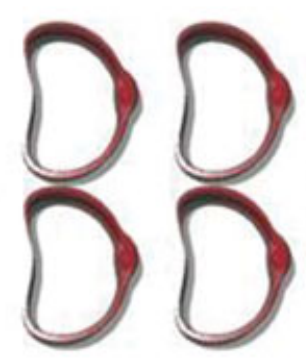

\section{Recipient capillaries (R)}

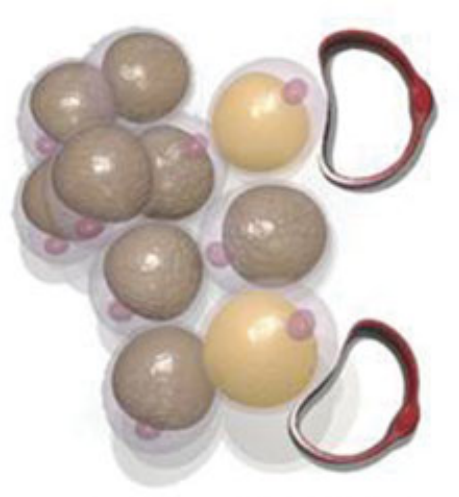

2 Surviving grafts (RG) 8 Necrotic grafts (G)

Fig. 2 The stoichiometry principle of fat grafting requires each fat droplet $(G)$ to pair with a recipient capillary vascular supply (R) in a $1: 1$ ratio for adequate revascularization. All unmatched fat tissue will not survive.

form a successful fat-recipient (GR) complex. The presence of more fat droplets than capillary recipient sites will lead to insufficient neoangiogenesis and is thought to be the reason behind fat resorption and necrosis. ${ }^{8}$ This concept, first described by Khouri and Khouri, is referred to as the stoichiometry principle of fat grafting (- Fig. 2), ${ }^{5,9}$ Ideally, there should be an optimal graft-recipient interface and good vascularity and oxygen tension of the recipient tissue., ${ }^{9,14}$

\section{Response to Injury and Mechanical Force}

When adipose tissue is injured, it undergoes degenerative changes that trigger the release of inflammatory and injuryrelated cytokines to promote wound healing. ASCs are stimulated by this process, proliferating and secreting secondary factors such as HGF and VEGF to regenerate adipose tissue and suppress fibrosis during the first week of injury. ${ }^{14,18}$ Bone marrow progenitor cells are also recruited and collaborate with ASCs to repair adipose tissue. ${ }^{14}$ Mechanical forces, both exogenous and endogenous, can affect the cellular function, survival, and growth of adipose tissue. ${ }^{14}$ Different fat graft harvesting techniques place different amounts of mechanical stress on adipocytes, and studies have demonstrated differences in cell viability and adipocyte functionality between techniques. ${ }^{4}$ Physical interactions with the extracellular matrix of adipose tissue can also influence stem cell behavior, and external tissue expansion has been shown experimentally to stimulate adipogenesis, resulting in a reversible enlargement of adipose tissue. ${ }^{14,17,19}$

\section{Graft Survival}

Currently, three theories have been put forth describing how fat grafts survive after avascular surgical implantation, and research suggests that each theory may play a role in the survival process. ${ }^{7}$ The theories are called the graft survival theory, the graft replacement theory, and the host cell replacement theory. 


\section{Graft Survival Theory}

This theory, first described by Peer et al, ${ }^{20}$ states that after surgical transfer, fat grafts initially survive through nutrient diffusion from the plasma until neovascularization from the recipient site occurs. ${ }^{7}$ Only those fat cells with sufficient new blood supply can survive. ${ }^{21}$ Smaller volume grafts may thus demonstrate better survival than larger volume grafts, as smaller volumes are better suited to achieve complete diffusion and perfusion.

\section{Graft Replacement Theory}

This theory states that very few donor adipocytes survive the grafting process; instead, grafted adipocytes are largely replaced by the donor ASCs that are concurrently transferred in the graft. ${ }^{7}$ It is the donor SVF that is responsible for adipogenesis and angiogenesis. ${ }^{7}$ Enrichment of fat grafts with SVF and ASCs has been investigated in multiple studies and has demonstrated significant increases in graft retention. ${ }^{22}$ Higher density fat grafts have also been shown to possess greater ASC concentrations and thus have increased survival rates compared with lower density grafts. ${ }^{23}$

\section{Host Cell Replacement Theory}

According to this theory, no grafted cells survive, and all the cells are replaced by recipient cells. ${ }^{7}$ Grafted cells necrotize and are replaced with fibrous tissue, new fat cells, and blood vessel ingrowth from the recipient tissue. ${ }^{7}$ Thus, the integrity and environment of the recipient site are major determinants of graft survival. In the past few decades, several methods to prepare the recipient site have been proposed, and fat grafting surgical outcomes have shown improvement as a result. ${ }^{24}$

\section{Three-Zone Survival Theory}

The survival of adipose tissue after autologous fat grafting can be further described by the three-zone survival theory established by Eto et al in 2012. ${ }^{25}$ This theory states that when the avascular fat is transferred, it can be divided into three cellular zones (-Fig. 3). ${ }^{5}$ The most peripheral zone is the survival zone, which is less than $300 \mu \mathrm{m}$ in thickness and contains adipocytes and ASCs that survive after grafting. 5,8,14 Directly underneath the survival zone is the regenerative zone, which is 600 to $1,200 \mu \mathrm{m}$ in thickness. ${ }^{5,9,14}$ Here, adipocytes die and get resorbed, but ASCs survive and regenerate into new adipocytes. ${ }^{5,8}$ The innermost zone is the central necrotic zone where no cells survive because of hypoxia. ${ }^{5}$ In this zone, no regeneration occurs and the dead space is either resorbed or filled with fibrosis. ${ }^{8}$ Based on the three-zone concept, the degree of survival of the regenerating zone contributes to the overall retained graft volume. ${ }^{1}$

After surgical transfer of the nonvascularized autologous fat tissue, the grafts are initially nourished through plasmatic diffusion from the surrounding host tissue for several days. ${ }^{8,14}$ Then, as early as 48 hours postimplantation, new blood vessels will begin supplying nutrients to the grafts. ${ }^{8,14}$ Revascularization occurs centripetally starting from the periphery and working its way to the center of the fat droplets. ${ }^{1}$ Until revascularization occurs, under severe ischemia, most adipocytes in the regenerative and necrotic zones will die within the first 24 hours of grafting and release inflammatory and injury-related factors. ${ }^{14,21}$ Among cellular components of adipose tissue, mature adipocytes are the most susceptible to ischemia and die first. ${ }^{14,25}$ If ischemia continues, vascular endothelial cells and blood-derived cells will die next. ${ }^{14}$ ASCs are relatively resistant to ischemia and can survive up to 3 days under severely ischemic conditions. ${ }^{14,25}$ During this time, ASCs undergo proliferation and adipogenesis as well as promote angiogenesis in response to signals from nearby dying cells to facilitate the repair process and restore the damaged tissue. ${ }^{14,25}$ Revascularization in the regenerating zone is thus improved by ASCs within these

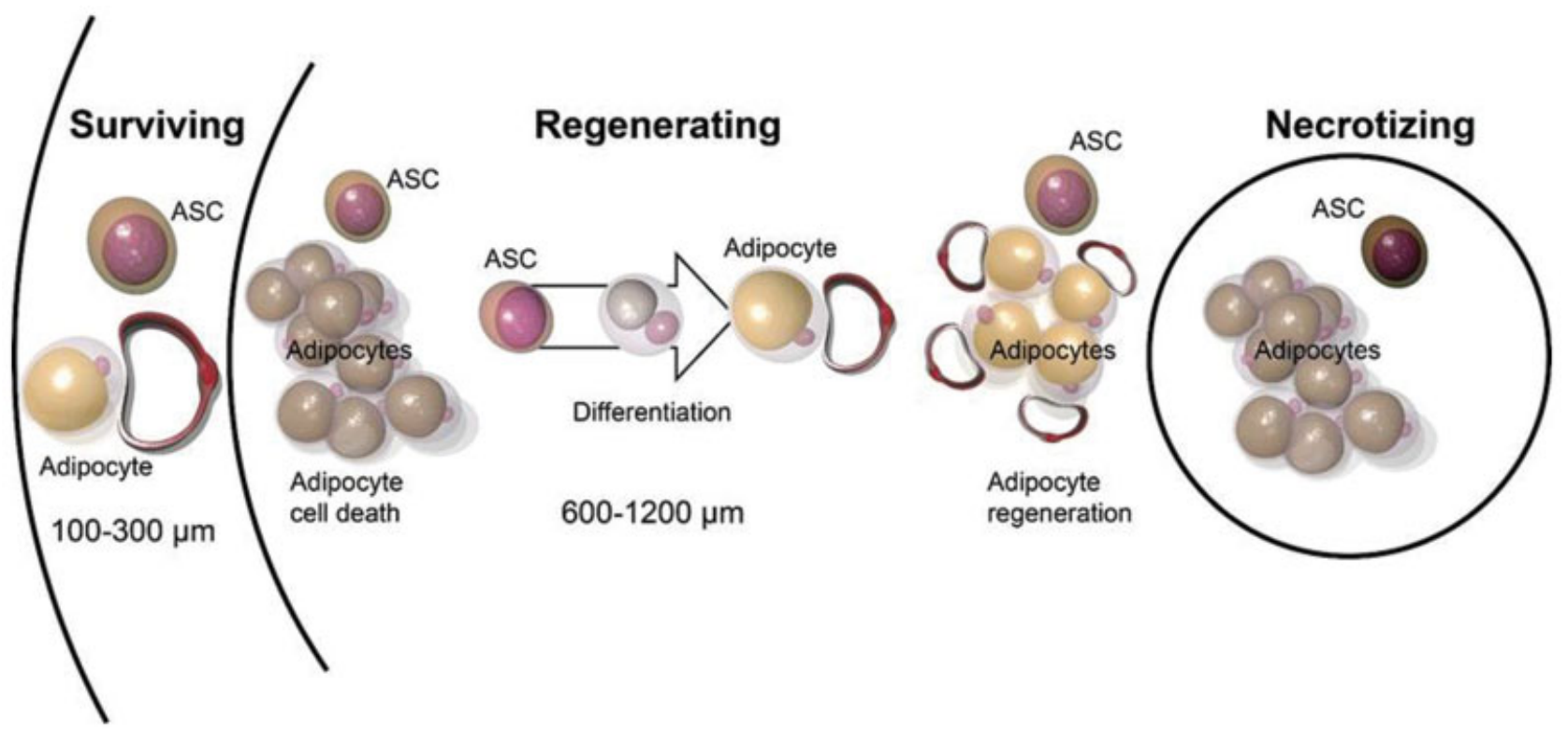

Fig. 3 Three-zone survival theory for fat graft. The avascular fat graft is composed of three zones: outermost survival zone, middle regenerative zone, and central necrotic zone. 
first 3 days following grafting. ${ }^{14}$ Adipogenesis from ASCs replaces dead adipocytes in the regenerative zone until 3 months following grafting. ${ }^{14,26}$ Interestingly, phagocytosis of dead adipocytes takes several weeks to months depending on tissue size; therefore, the volume of grafted fat remains unchanged for the first 4 weeks despite significant adipocyte death. $^{14,26}$

The "acute" regenerative and adipogenic phase of fat graft survival is completed by 3 months and is followed by a "chronic" stabilizing process that can persist for up to additional 9 months; the total remodeling process following fat grafting can thus take up to a year (-Fig. 4). ${ }^{14}$ During this "chronic" phase, lipid absorption continues without compensatory ASC regeneration. ${ }^{14}$ Any remaining dead adipocytes in the regenerative or central necrotic zones will either be resorbed, undergo fibrogenesis, or form oil cysts. ${ }^{14}$ The speed of resorption depends on the diameter of the lipid droplets. ${ }^{21}$ Droplets $<8 \mathrm{~mm}$ in diameter can be resorbed relatively quickly and replaced by fibrosis. ${ }^{14}$ Droplets $>8 \mathrm{~mm}$ in diameter are resorbed more slowly, and permanent oil cysts form before these large droplets can be completely resorbed, leading to chronic inflammation and calcification over time. ${ }^{14,26,27}$

The balance between the rates of lipid resorption, necrosis, and successful adipocyte replacement plays a prominent role in determining final graft volume retention. ${ }^{14,21}$ If the graft volume is small and lipid resorption and adipocyte regeneration are complete within the 3-month time period, then the final volume of the graft is unlikely to change significantly after 3 months. ${ }^{14,26}$ However, if the graft volume is large, then many large droplets will be incompletely absorbed by 3 months and will continue to undergo absorption without compensatory adipogenesis for up to a year, resulting in clinically significant volume reduction. ${ }^{14}$ The ultimate graft volume retained is influenced by many addi- tional factors such as graft microenvironment, vascularity, processing techniques, and postoperative care. ${ }^{14}$

\section{Large-Volume Fat Grafts}

In order for fat cells to survive, oxygen must be able to diffuse through the fat droplet to provide nourishment. ${ }^{5}$ Thus, only microdroplets with a maximum regenerative zone depth of $1.6 \mathrm{~mm}$ are able to fully revascularize and survive without developing a central necrotic zone. ${ }^{5,9}$ When trying to graft larger fat volumes, a high number of small individual grafts should be deposited in different tissue planes to prevent droplet coalescence. ${ }^{5,9}$ If allowed to coalesce, larger grafts will exhibit high levels of necrosis, liquefaction, and cyst formation due to poor nutrient diffusion from plasma and inadequate neovascularization to the central zone. ${ }^{8}$

\section{Browning of Adipose Tissue}

One interesting phenomenon that has recently been described is the "browning" of WAT. In addition to the commonly known WAT and BAT, adults have also been shown to have beige (or brite) adipose tissue. This beige adipose tissue is an inducible brownlike adipose tissue that, when found among WAT, causes WAT to have BAT-like metabolic characteristics. This phenomenon is called "browning." In recent experiments, Hoppela et al demonstrated that when subcutaneous WAT was grafted into the muscle tissue, the graft exhibited "browning," ${ }^{6}$ that is, transfer of metabolically inactive WAT into a new environment changed the metabolic activity of the grafted tissue to resemble the metabolic activity of the recipient site. This study showed that different depots of fat have different inherent metabolic activity and that adipose tissue can undergo metabolic changes upon grafting to certain new locations. This metabolic change could impact graft site glucose tolerance, metabolism, and body weight. $^{6}$

\section{Acute Repair Phase Chronic Resorption Phase}

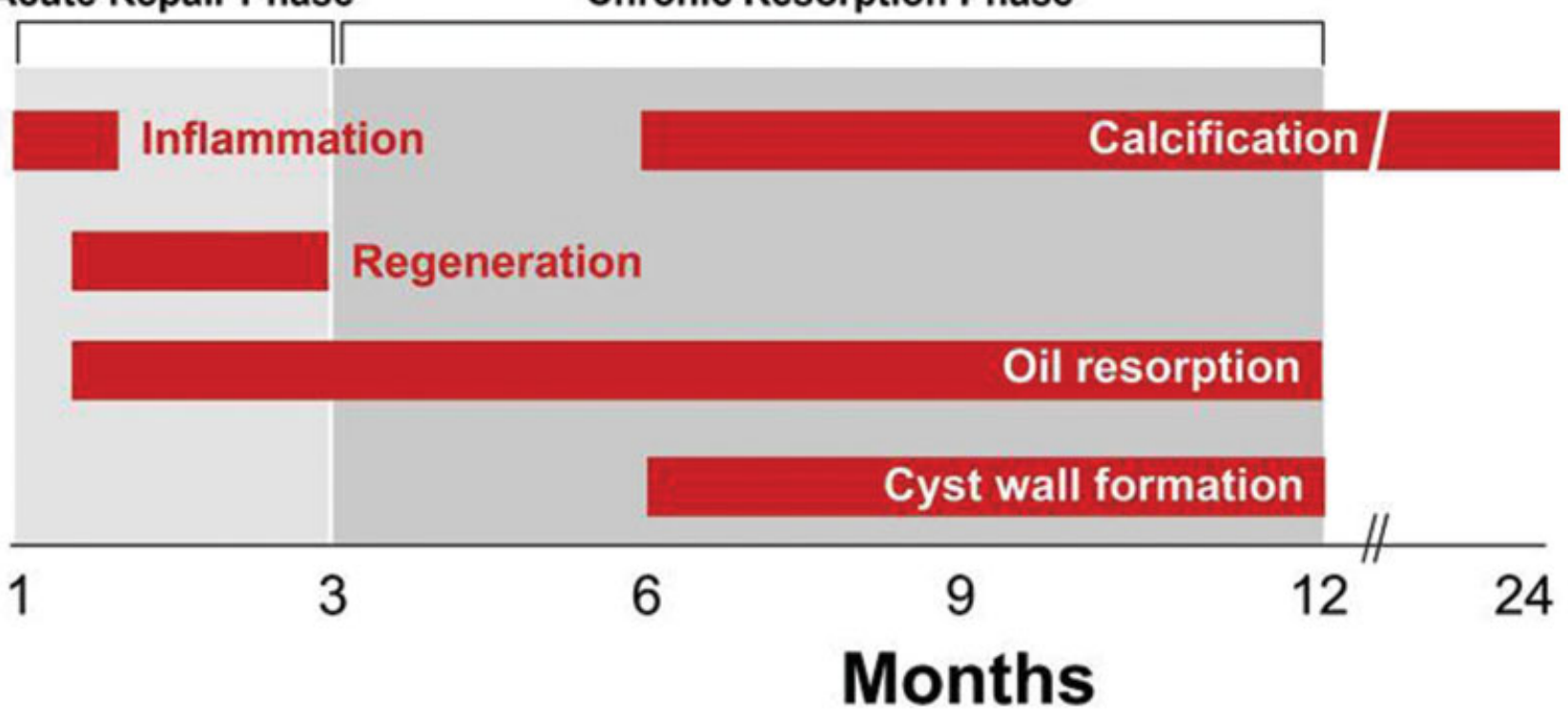

Fig. 4 Fat graft undergoes acute repair phase until 3 months and chronic absorption phase from 3 to 12 months. 


\section{Conclusion}

Autologous fat grafting is a common technique used by many plastic surgeons in a variety of aesthetic and reconstructive procedures. Predicting fat graft volume retention remains a challenge and largely depends on the balance between regeneration and resorption of adipose tissue, which is affected by many factors including graft size, procedure techniques, and graft microenvironment. While the exact mechanism underlying the survival of adipose tissue after fat grafting is not entirely understood, many studies have investigated this topic and several well-supported theories have been proposed. Understanding the biology and scientific principles behind adipose tissue can give us a better insight into ideal fat grafting methods that can produce longlasting and desirable results.

\section{Conflicts of Interest}

The authors have no conflicts to disclose.

\section{Acknowledgments}

The authors would like to thank Scott Holmes, CMI, a member of the Michael E. DeBakey Department of Surgery, Baylor College of Medicine, for his graphic assistance during the preparation of this manuscript.

\section{References}

1 Bellini E, Grieco MP, Raposio E. The science behind autologous fat grafting. Ann Med Surg (Lond) 2017;24(October):65-73

2 Zielins ER, Brett EA, Longaker MT, Wan DC. Autologous fat grafting: the science behind the surgery. Aesthet Surg J 2016;36 (04):488-496

3 Agarwal K, Mistry M. Autologous fat transfer for breast surgery. $\mathrm{Br}$ J Hosp Med (Lond) 2017;78(08):448-452

4 Strong AL, Cederna PS, Rubin JP, Coleman SR, Levi B. The current state of fat grafting: a review of harvesting, processing, and injection techniques. Plast Reconstr Surg 2015;136(04):897-912

5 Abu-Ghname A, Perdanasari AT, Reece EM. Principles and applications of fat grafting in plastic surgery. Semin Plast Surg 2019;33 (03):147-154

6 Hoppela E, Grönroos TJ, Saarikko AM, et al. Fat grafting can induce browning of white adipose tissue. Plast Reconstr Surg Glob Open 2018;6(06):e1804

7 Doornaert M, Colle J, De Maere E, Declercq H, Blondeel P. Autologous fat grafting: latest insights. Ann Med Surg (Lond) 2018;37 (37): $47-53$

8 Liao HT, Marra KG, Rubin JP. Application of platelet-rich plasma and platelet-rich fibrin in fat grafting: basic science and literature review. Tissue Eng Part B Rev 2014;20(04):267-276

9 Khouri RK Jr, Khouri RK. Current clinical applications of fat grafting. Plast Reconstr Surg 2017;140(03):466e-486e
10 Saely CH, Geiger K, Drexel H. Brown versus white adipose tissue: a mini-review. Gerontology 2012;58(01):15-23

11 Stanford KI, Middelbeek RJW, Goodyear LJ. Exercise effects on white adipose tissue: beiging and metabolic adaptations. Diabetes 2015;64(07):2361-2368

12 Bucky LP, Percec I. The science of autologous fat grafting: views on current and future approaches to neoadipogenesis. Aesthet Surg J 2008;28(03):313-321, quiz 322-324

13 Cartwright MJ, Tchkonia T, Kirkland JL. Aging in adipocytes: potential impact of inherent, depot-specific mechanisms. Exp Gerontol 2007;42(06):463-471

14 Mashiko T, Yoshimura K. How does fat survive and remodel after grafting? Clin Plast Surg 2015;42(02):181-190

15 Suga H, Eto H, Aoi N, et al. Adipose tissue remodeling under ischemia: death of adipocytes and activation of stem/progenitor cells. Plast Reconstr Surg 2010;126(06):1911-1923

16 Ogura F, Wakao S, Kuroda Y, et al. Human adipose tissue possesses a unique population of pluripotent stem cells with nontumorigenic and low telomerase activities: potential implications in regenerative medicine. Stem Cells Dev 2014;23(07):717-728

17 Kato H, Suga H, Eto H, et al. Reversible adipose tissue enlargement induced by external tissue suspension: possible contribution of basic fibroblast growth factor in the preservation of enlarged tissue. Tissue Eng Part A 2010;16(06):2029-2040

18 Eto $\mathrm{H}$, Suga $\mathrm{H}$, Inoue $\mathrm{K}$, et al. Adipose injury-associated factors mitigate hypoxia in ischemic tissues through activation of adipose-derived stem/progenitor/stromal cells and induction of angiogenesis. Am J Pathol 2011;178(05):2322-2332

19 Khouri RK, Schlenz I, Murphy BJ, Baker TJ. Nonsurgical breast enlargement using an external soft-tissue expansion system. Plast Reconstr Surg 2000;105(07):2500-2512, discussion 2513-2514

20 Peer LA. Loss of weight and volume in human fat grafts with postulation of a "Cell Survival Theory". Plast Reconstr Surg 1950; 5:217-230

21 Pu LLQ. Mechanisms of fat graft survival. Ann Plast Surg 2016;77 (00, Suppl 1):S84-S86

22 Rasmussen BS, Lykke Sørensen C, Vester-Glowinski PV, et al. Effect, feasibility, and clinical relevance of cell enrichment in large volume fat grafting: a systematic review. Aesthet Surg J 2017;37(3, suppl_3):S46-S58

23 Allen RJ Jr, Canizares O Jr, Scharf C, et al. Grading lipoaspirate: is there an optimal density for fat grafting? Plast Reconstr Surg 2013;131(01):38-45

24 Oranges CM, Striebel J, Tremp M, et al. The preparation of the recipient site in fat grafting: a comprehensive review of the preclinical evidence. Plast Reconstr Surg 2019;143(04):1099-1107

25 Eto $\mathrm{H}$, Kato $\mathrm{H}$, Suga $\mathrm{H}$, et al. The fate of adipocytes after nonvascularized fat grafting: evidence of early death and replacement of adipocytes. Plast Reconstr Surg 2012;129(05):1081-1092

26 Yoshimura K, Eto H, Kato H, Doi K, Aoi N. In vivo manipulation of stem cells for adipose tissue repair/reconstruction. Regen Med 2011;6(6, Suppl):33-41

27 Mineda K, Kuno S, Kato $\mathrm{H}$, et al. Chronic inflammation and progressive calcification as a result of fat necrosis: the worst outcome in fat grafting. Plast Reconstr Surg 2014;133(05): 1064-1072 